\begin{tabular}{lll}
\hline VOL. 79 & 1999 & NO. 2 \\
\hline
\end{tabular}

\title{
ARITHMETIC FUNCTIONS AND WEIGHTED AVERAGES
}

\author{
BY \\ JEAN-MARIE DE KONINCK AND \\ JACQUES GRAH (SAINTE-FOY, QUÉBEC)
}

1. Introduction. It is well known that any arithmetical function $f$ can be written as

$$
f(n)=\sum_{d \mid n} g(d)
$$

with $g$ defined by $g=\mu * f$, where $*$ is the Dirichlet convolution and $\mu$ denotes the Möbius function: $\mu(1)=1, \mu(n)=0$ if $p^{2} \mid n$ for some prime $p$, and $\mu(n)=(-1)^{r}$ if $n=q_{1} \ldots q_{r}$ is the canonical prime-power factorization of $n$.

Another way of expressing a number-theoretic function $f$ is to restrict the sum in (1.1) to what is commonly called the unitary divisors $d$ of $n$, that is,

$$
f(n)=\sum_{\begin{array}{c}
d \mid n \\
(d, n / d)=1
\end{array}} g(d) .
$$

One can also consider the classical "weighted averages", based on (1.1) and (1.2). Given an arithmetic function $f$, define the functions $f_{1}, f_{2}$ and $f_{3}$ by

$$
\begin{gathered}
f_{1}(n):=\frac{1}{\tau(n)} \sum_{d \mid n} f(d), \quad f_{2}(n):=\frac{1}{2^{\omega(n)}} \sum_{\substack{d \mid n \\
(d, n / d)=1}} f(d), \\
f_{3}(n):=\frac{1}{2^{\omega(n)}} \sum_{d \mid n} \mu^{2}(d) f(d),
\end{gathered}
$$

where $\tau(n)$ and $\omega(n)$ stand respectively for the number of positive divisors

1991 Mathematics Subject Classification: 11A25, 11N37.

Key words and phrases: arithmetic functions, weighted averages, mean value of arithmetic functions.

Research partially supported by grants from NSERC of Canada and FCAR of Québec. 
and for the number of distinct prime factors of $n$. Each of these three functions represents an "average" value of $f(d)$ as $d$ runs through a subset of divisors $d$ of an integer $n$.

Recently, we studied extensively the arithmetic properties of these averaging functions (see De Koninck and Grah [5]). We showed, amongst other things, that, although it is not the case for the functions $f$ defined by (1.1) and (1.2), $f_{1}, f_{2}$ and $f_{3}$ "inherit" the arithmetic properties of $f$. In fact, we proved that if $f$ is additive, then $f_{1}, f_{2}$ and $f_{3}$ are additive as well and, conversely, if $f_{1}$ or $f_{2}$ is additive, then so is $f$. Moreover, mean value theorems related to $f_{1}, f_{2}$ and $f_{3}$ were obtained. For instance, we showed that, for a wide class of arithmetic additive functions $f$, the averaging functions $f_{1}$ and $f_{2}$ have a mean value if and only if $f$ has a mean value.

In this paper, given a real-valued arithmetic function $f$, we show that under certain conditions, the corresponding functions $f_{1}, f_{2}$ and $f_{3}$ as well as other weighted averages of arithmetic functions satisfy mean value theorems, and from these we derive known and also new results.

2. Motivation and necessary background. Let $s$ be a fixed real number. Using results of De Koninck and Grah [5] (théorème 5.2), one can prove that, given any arithmetic function $f$, there exist uniquely defined arithmetic functions $\bar{f}_{s}$ and $\widetilde{f}_{s}$ such that $f$ admits the representations

$$
f(n)=\frac{1}{n^{s}} \sum_{d \mid n} \bar{f}_{s}(d) \phi_{s}(d)
$$

and

$$
f(n)=\frac{\phi_{s}(n)}{n^{s}} \sum_{\substack{d \mid n \\(d, n / d)=1}} \frac{\widetilde{f}_{s}(d)}{\phi_{s}(\delta(d))},
$$

where $\delta(n)$ represents the largest squarefree divisor of $n$, i.e. $\delta(1)=1$ and $\delta(n)=q_{1} q_{2} \ldots q_{r}$ if $n=q_{1}^{\alpha_{1}} q_{2}^{\alpha_{2}} \ldots q_{r}^{\alpha_{r}}$ is the canonical decomposition of $n$ (sometimes $\delta(n)$ is referred to as the core function), and where $\phi_{s}$ is the extension to real numbers $s$ of the Jordan totient function, that is,

$$
\phi_{s}(n):=n^{s} \prod_{p \mid n}\left(1-\frac{1}{p^{s}}\right)=n^{s} \sum_{d \mid n} \frac{\mu(d)}{d^{s}},
$$

the product ranging over the primes $p$ dividing $n$; for convenience, we set $\phi_{0}(n)=1(n)$, where $1(n)=1$ for all integers $n \geq 1$.

Representations (2.1) and (2.2) can easily be obtained for any given arithmetic function $f$. Indeed, in De Koninck and Grah [5] (théorème 5.2), we gave an extension of the three equalities in (1.3); hence, by restricting $*_{A}$ to Dirichlet convolution, and replacing the functions $g(n)$ and $U(n)$, 
involved in that result, by $\mu(n)$ and $n^{s}(s \in \mathbb{R})$ respectively, one obtains (2.1) by the Möbius inversion formula. Similarly, (2.2) is a restriction to unitary convolution, where $g$ and $U$ are respectively replaced by $(-1)^{\omega(n)}$ and $n^{s} / \phi_{s}(n)(s \in \mathbb{R})$.

Before moving on, we give three examples:

(a) Set $s=1 / 2$ and let $f$ be the strongly multiplicative function (that is, one which satisfies $f\left(p^{\alpha}\right)=f(p)$ for all primes $p$ and all integers $\alpha \geq 1$ ) defined by $f(n)=\prod_{p \mid n}(1-2 / \sqrt{p})$; we immediately compute $\widetilde{f}_{1 / 2}\left(p^{\alpha}\right)=-1$ (for any prime $p$ and any positive integer $\alpha$ ), so that to $f(n)=\prod_{p \mid n}(1-$ $2 / \sqrt{p})$ corresponds $\widetilde{f}_{1 / 2}(n)=(-1)^{\omega(n)}$.

(b) Set $s=1$ and let $f(n)=\phi(n) / n$, where $\phi$ stands for the Euler totient function; then we obtain $\widetilde{f}_{1}\left(p^{\alpha}\right)=0$ for any $\alpha \geq 1$, so that to $\phi(n) / n$ corresponds

$$
\widetilde{f}_{1}(n)=E(n):= \begin{cases}1 & \text { if } n=1, \\ 0 & \text { if } n>1 .\end{cases}
$$

(c) Let $f(n)=\omega(n)$; then $\bar{\omega}_{s}(n)=\sum_{p|| n} \frac{p^{s}}{p^{s}-1}+\sum_{p^{2} \mid n} 1$, while $\widetilde{\omega}_{s}(n)=$ $\sum_{p \mid n} p^{s}$.

Both functions $\bar{f}_{s}$ and $\widetilde{f}_{s}$ (for $s \neq 0$ ) have interesting properties. In fact, by inversion, we see that both are weighted averages of the values of $f(d)$ as $d$ runs through particular sets of divisors of $n$. The former is an averaging over all divisors, the weight at $f(d)$ being $\mu(n / d) d^{s}$, and the latter is an averaging over unitary divisors with $1 / \phi_{s}(\delta(n))$ as total weight. These two functions constitute the other class of weighted averages we study in this paper. From the behaviour of series related to the weighted average $\bar{f}_{s}$, we prove the existence of a constant $C_{s}=C_{s}(f)$ such that the number-theoretic function $C_{s} / n^{s}$ is a mean value of $f$.

For instance, consider the function

$$
f(n)= \begin{cases}\sum_{p \mid n} \frac{\log p}{p} \prod_{\substack{q \mid n \\ q \neq p}}\left(1-q^{-1}\right) & \text { for } n>1, \\ 1 & \text { for } n=1,\end{cases}
$$

where $p$ and $q$ denote primes. The mean value of $f$ is rather difficult to obtain directly; however, we easily deduce the equality

$$
\lim _{x \rightarrow \infty} x^{-1} \sum_{n \leq x} f(n)=\frac{1}{\zeta(2)} \sum_{p} \frac{\log p}{p^{2}-1},
$$

as an immediate consequence of an asymptotic mean value result we prove.

Given an arithmetic function $g$, we say that $g$ belongs to the class $\mathcal{W}$ if the series $\sum_{n=1}^{\infty}(\mu * g)(n) / n$ is absolutely convergent. For example, the 
right-hand side of (2.2) may be rewritten as

$$
\frac{\phi_{s}(n)}{n^{s}} \sum_{\substack{d \mid n \\
(d, n / d)=1}} \frac{\tilde{f}_{s}(d)}{\phi_{s}(\delta(d))}=\sum_{\begin{array}{c}
d \mid n \\
(d, n / d)=1
\end{array}} \frac{\tilde{f}_{s}(d)}{\delta(d)^{s}} \cdot \frac{\phi_{s}(n / d)}{(n / d)^{s}},
$$

where the last sum is the unitary convolution $\left(*_{\mathrm{u}}\right)$ of the functions $\phi_{s}(n) / n^{s}$ and $\widetilde{f}_{s}(n) / \delta(n)^{s}$. Then, for $s>0$, we see that $\phi_{s}(n) / n^{s} \in \mathcal{W}$, since $\phi_{s}(n) / n^{s}$ $=\sum_{d \mid n} \mu(d) / d^{s}$ and the series $\sum_{n=1}^{\infty} \mu(n) / n^{s+1}$ converges absolutely for $s>0$.

Now consider the right-hand side of $(2.5)$ in which we replace $\phi_{s}(n) / n^{s}$ by a function $g \in \mathcal{W}$ and $\widetilde{f}_{s}(n) / \delta(n)^{s}$ by a function $h$. We then obtain mean value results related to $h *_{\mathrm{u}} g$. In particular, for $\sigma_{s}^{*}(n)$, the sum of the $s$ th powers of unitary divisors of $n$, it will follow that, as $x \rightarrow \infty$,

$$
\frac{1}{x^{s+1}} \sum_{n \leq x} \sigma_{s}^{*}(n)=\frac{\zeta(s+1)}{(1+s) \zeta(s+2)} .
$$

Our goal is to investigate the existence of the mean value of some classes of weighted averages of functions as well as their asymptotic mean. Our results are based either on the behaviour of the averaging functions themselves or on the behaviour of the functions from which these averages stem. Even if their proofs are elementary, the results we state in the next section have certain importance of their own, as they deal with weighted averages of wide classes of functions known for their erratic behaviour. In several cases, we provide an example which reveals that importance.

3. Statement of the main results. Our first three results deal with the estimation of the size, in average, of weighted averages of number-theoretic functions.

THEOREM 1. Let $f$ be an arithmetic function for which $\sum_{n=1}^{\infty}|f(n)| / n$ is convergent. Moreover assume that, as $x \rightarrow \infty$,

$$
\sum_{n>x} \frac{f^{2}(n)}{n}=o\left(\frac{1}{\log ^{1 / 4} x}\right) .
$$

Then there exists a constant $A$ such that, as $x \rightarrow \infty$,

$$
\sum_{n \leq x}\left(\frac{1}{\tau(n)} \sum_{d \mid n} f(d)\right)=(A+o(1)) \frac{x}{\sqrt{\log x}},
$$

where

$$
A:=\frac{\alpha}{\sqrt{\pi}} \sum_{n=1}^{\infty} \frac{f(n) u(n)}{n}
$$


with $u$ being the multiplicative function defined by

and

$$
u\left(p^{r}\right):=\left(\sum_{i=0}^{\infty} \frac{p^{-i}}{i+r+1}\right)\left(\sum_{i=0}^{\infty} \frac{p^{-i}}{i+1}\right)^{-1}
$$

$$
\alpha:=\prod_{p} \sqrt{p(p-1)} \log \frac{p}{p-1}=0.969 \ldots
$$

THEOREM 2. Let $f$ be an arithmetic function for which $\sum_{n=1}^{\infty}|f(n)| / n$ is convergent. Moreover assume that, as $x \rightarrow \infty$,

$$
\sum_{n>x} \frac{f^{2}(n)}{n}=o\left(\frac{1}{\log ^{5 / 4} x}\right)
$$

Then, as $x \rightarrow \infty$,

$$
\sum_{n \leq x}\left(\frac{1}{2^{\omega(n)}} \sum_{\substack{d \mid n \\(d, n / d)=1}} f(d)\right)=(B+o(1)) \frac{x}{\sqrt{\log x}}
$$

where

$$
B:=\frac{\beta}{\sqrt{\pi}} \sum_{n=1}^{\infty} \frac{f(n)}{n 2^{\omega(n)}} \prod_{p \mid n}\left(1-\frac{1}{2 p-1}\right)
$$

with

$$
\beta:=\prod_{p}\left(1+\frac{1}{4 p(p-1)}\right)^{1 / 2}=1.969 \ldots
$$

In particular, as $x \rightarrow \infty$,

$$
\sum_{n \leq x}\left(\frac{1}{2^{\omega(n)}} \sum_{\substack{d \mid n \\ \text { squarefree }}} f(d)\right)=(D+o(1)) \frac{x}{\sqrt{\log x}},
$$

where

$$
D:=\frac{\beta}{\sqrt{\pi}} \sum_{n=1}^{\infty} \frac{\mu^{2}(n) f(n)}{n 2^{\omega(n)}} \prod_{p \mid n}\left(1+\frac{1}{2 p-1}\right) .
$$

TheOREM 3. Let $f$ be a multiplicative arithmetic function and let $h:=$ $\mu f * 1$. Assume that the series

$$
\sum_{n=1}^{\infty} \frac{\mu(n) h(n)}{n \tau(n)}=\sum_{n=1}^{\infty} \frac{\mu(n) h(n)}{n 2^{\omega(n)}}=\sum_{n=1}^{\infty} \frac{\mu(n) h(n)}{n 2^{\Omega(n)}}
$$

is absolutely convergent. Then

$$
\lim _{x \rightarrow \infty} x^{-1} \sum_{n \leq x}\left(\frac{1}{2^{\omega(n)}} \sum_{\substack{d \mid n \\ d \text { squarefree }}} f(d)\right)=\sum_{n=1}^{\infty} \frac{\mu(n) h(n)}{n \tau(n)}=\prod_{p}\left(1-\frac{h(p)}{2 p}\right) .
$$


In particular, when $f$ is strongly multiplicative,

$$
\begin{aligned}
& \lim _{x \rightarrow \infty} x^{-1} \sum_{n \leq x}\left(\frac{1}{2^{\omega(n)}} \sum_{\substack{d \mid n \\
(d, n / d)=1}} f(d)\right) \\
&=\lim _{x \rightarrow \infty} x^{-1} \sum_{n \leq x}\left(\frac{1}{2^{\omega(n)}} \sum_{\substack{d \mid n \\
\text { squarefree }}} f(d)\right) .
\end{aligned}
$$

EXAMPLE. If $f(n)=\phi(n) / n$, which is a strongly multiplicative function, then $h(n)=1 / \delta(n)$ and since $\sum_{n=1}^{\infty} \mu(n) /(n \delta(n) \tau(n))$ is an absolutely convergent series, (3.6) and (3.7) yield

$$
\begin{aligned}
\lim _{x \rightarrow \infty} x^{-1} \sum_{n \leq x} f_{2}(n) & =\lim _{x \rightarrow \infty} x^{-1} \sum_{n \leq x} f_{3}(n) \\
& =\prod_{p}\left(1-\frac{1}{2 p^{2}}\right)=0.789 \ldots,
\end{aligned}
$$

where $f_{2}$ and $f_{3}$ are defined in (1.3), thereby providing the average behaviour of $\phi(d) / d$ as $d$ runs over the squarefree divisors of $n$.

The next theorem uses (2.1) in establishing the mean value of a function $f$, based on the behaviour of the series of its weighted average $\bar{f}_{s}$. In fact, Theorem 4 establishes a mean value and an asymptotic mean for classes of functions $f$ whose Dirichlet convolution with the function $\mu(n) / n^{s}$, according to the parameter $s$, is the general term of an absolutely convergent series.

TheOREM 4. For a fixed real number $s$, let an arithmetic function $f$ be expressed in the form

$$
f(n)=\frac{1}{n^{s}} \sum_{d \mid n} \bar{f}_{s}(d) \phi_{s}(d)
$$

(i) If $\sum_{n=1}^{\infty} \bar{f}_{1}(n) \phi(n) / n$ is absolutely convergent, then as $x \rightarrow \infty$,

$$
\sum_{n \leq x} f(n)=\left(c_{1}+o(1)\right) \log x, \quad \text { where } \quad c_{1}=\sum_{n=1}^{\infty} \frac{\bar{f}_{1}(n) \phi(n)}{n} .
$$

(ii) If $\sum_{n=1}^{\infty} \bar{f}_{s}(n) \phi(n) / n$ is absolutely convergent for $0 \leq s<1$, then as $x \rightarrow \infty$,

$$
\sum_{n \leq x} f(n)=(1+o(1)) \frac{1}{1-s} \sum_{n=1}^{\infty} \frac{\bar{f}_{s}(n) \phi_{s}(n)}{n} \cdot x^{1-s} .
$$

(iii) If $\sum_{n=1}^{\infty} \bar{f}_{s}(n) \phi_{s}(n) / n^{s}$ is absolutely convergent for $s>1$, then as 
$x \rightarrow \infty$

$$
\sum_{n \leq x} f(n)=\zeta(s) \sum_{n=1}^{\infty} \frac{\bar{f}_{s}(n) \phi_{s}(n)}{n^{s}}+o(1),
$$

where $\zeta(s)$ stands for the Riemann zeta function.

(iv) Let $r=-s>0$. If $\sum_{n=1}^{\infty} \bar{f}_{r}(n) \delta(n)^{r} / n^{r+1}$ is absolutely convergent, then as $x \rightarrow \infty$,

$$
\begin{gathered}
\sum_{n \leq x} f(n)=\left(c_{r}+o(1)\right) \frac{x^{r+1}}{r+1}, \quad \text { where } \\
c_{r}:=\sum_{n=1}^{\infty} \frac{\bar{f}_{r}(n)(-1)^{\omega(n)} \phi_{r}(\delta(n))}{n^{r+1}} .
\end{gathered}
$$

Remarks. 1. The asymptotic estimate provided by (3.9) remains true if the series in (ii) is replaced by $\sum_{n=1}^{\infty} \bar{f}_{s}(n) \phi_{s}(n) / n$. In this particular case, setting $s=0$ in equation (3.9) yields a well-known result (see [4], Proposition 4), which is sometimes referred to as Wintner's Theorem (see [9]). The same can be said about (3.11), the series in (iv) and the one defining $c_{r}$.

2. It is noteworthy that in each of the four cases of Theorem 4, there exists a constant $C_{s}=C_{s}(f)$ such that

$$
\sum_{n \leq x} f(n) \sim C_{s} \sum_{n \leq x} \frac{1}{n^{s}}
$$

that is, $C_{s} / n^{s}$ is a mean value of $f$.

Our next result shows that, for a certain class of arithmetic functions written using (2.2), the asymptotic mean value is closely linked to the Riemann zeta function.

THEOREM 5. Let $s$ be a positive number and $f$ an arithmetic function whose representation is given by

$$
f(n)=\frac{\phi_{s}(n)}{n^{s}} \sum_{\substack{d \mid n \\(d, n / d)=1}} \frac{\widetilde{f}_{s}(d)}{\phi_{s}(\delta(d))},
$$

where $\widetilde{f}_{s}$ is bounded. Then, as $x \rightarrow \infty$,

$$
\sum_{n \leq x} f(n)=\left(\frac{d_{s}}{\zeta(s+1)}+o(1)\right) x
$$


where

$$
d_{s}:=\sum_{n=1}^{\infty} \frac{\widetilde{f}_{s}(n) n^{s-1} \phi(n)}{\delta(n)^{s} \phi_{s+1}(n)}=\sum_{n=1}^{\infty} \frac{\widetilde{f}_{s}(n)}{n \delta(n)^{s}} \prod_{p \mid n}\left(1-\frac{p^{s}-1}{p^{s+1}-1}\right) .
$$

REMARKS. Since $\widetilde{f}_{s}$ is bounded, for $s>0$ there exists a positive constant $c$ such that for all $n \geq 1$,

$$
\frac{\widetilde{f}_{s}(n) n^{s-1} \phi(n)}{\delta(n)^{s} \phi_{s+1}(n)}=\frac{\widetilde{f}_{s}(n)}{n \delta(n)^{s}} \prod_{p \mid n}\left(1-\frac{p^{s}-1}{p^{s+1}-1}\right) \leq \frac{c}{n \delta(n)^{s}} .
$$

Now, $\sum_{n=1}^{\infty} 1 /\left(n \delta(n)^{s}\right)$ is convergent for $s>0$ since its Euler product representation is

$$
\prod_{p}\left(1+\frac{1}{p^{s}(p-1)}\right)
$$

It follows that

$$
\sum_{n=1}^{\infty} \frac{\widetilde{f}_{s}(n) n^{s-1} \phi(n)}{\delta(n)^{s} \phi_{s+1}(n)}=\sum_{n=1}^{\infty} \frac{\widetilde{f}_{s}(n)}{n \delta(n)^{s}} \prod_{p \mid n}\left(1-\frac{p^{s}-1}{p^{s+1}-1}\right)
$$

is absolutely convergent.

2. From the above remark, we note that (3.12) holds if the boundedness of $\widetilde{f}_{s}$ is replaced by the absolute convergence of the series defining $d_{s}$.

Example. Consider the function $f$ defined by (2.4). Then for $s=1$, one easily obtains $\widetilde{f}_{1}(n)=\Lambda(n)$, where $\Lambda(n)$ is the von Mangoldt function which is null unless $n$ is a power of a prime $p$, in which case $\Lambda(n)=\log p$. Clearly, $\Lambda$ is unbounded. Nevertheless, it follows from Theorem 5, taking into account the second remark above, that

$$
\begin{aligned}
\lim _{x \rightarrow \infty} x^{-1} \sum_{n \leq x} f(n) & =\frac{1}{\zeta(2)} \sum_{n=1}^{\infty} \frac{\Lambda(n)}{n \delta(n)} \prod_{p \mid n}\left(1-\frac{1}{p+1}\right) \\
& =\frac{1}{\zeta(2)} \sum_{p} \frac{\log p}{p^{2}-1}=0.346 \ldots
\end{aligned}
$$

Theorem 6. Let $f$ be an arithmetic function defined by

$$
f(n)=\sum_{\substack{d \mid n \\(d, n / d)=1}} h(d) g(n / d)
$$

for a given $g \in \mathcal{W}$. If $\sum_{n=1}^{\infty} h(n) / n$ is absolutely convergent, then

$$
\lim _{x \rightarrow \infty} x^{-1} \sum_{n \leq x} f(n)=\sum_{n=1}^{\infty} \frac{h(n)}{n} \sum_{d \mid n} \frac{\mu(d)}{d} \sum_{r \mid d} \frac{\phi(r)}{r} \sum_{m=1}^{\infty} \frac{(\mu * g)(r m)}{m} .
$$


REMARK. It is interesting to observe that for the particular case $g=1$, we have $\mu * g=E$ (where 1 and $E$ are the functions defined in $\S 2$ ), so that

$$
\sum_{d \mid n} \frac{\mu(d)}{d} \sum_{r \mid d} \frac{\phi(r)}{r} \sum_{m=1}^{\infty} \frac{(\mu * g)(r m)}{m}=\frac{\phi(n)}{n}
$$

and (3.14) becomes

$$
\lim _{x \rightarrow \infty} x^{-1} \sum_{n \leq x} f(n)=\sum_{n=1}^{\infty} \frac{h(n) \phi(n)}{n^{2}},
$$

which was established in [7].

COROLlary 7. If $s$ is a positive number and $g \in \mathcal{W}$, then as $x \rightarrow \infty$,

$$
\lim _{x \rightarrow \infty} x^{-1} \sum_{n \leq x} \sum_{\substack{d \mid n \\(d, n / d)=1}} \frac{g(n / d)}{d^{s}}=A_{s},
$$

where

$$
A_{s}:=\sum_{n=1}^{\infty} \frac{1}{n^{s+1}} \sum_{d \mid n} \frac{\mu(d)}{d} \sum_{r \mid d} \frac{\phi(r)}{r} \sum_{m=1}^{\infty} \frac{(\mu * g)(r m)}{m} .
$$

ExAmple. For $s>0$, if we set $g=1$ in $(3.15)$, then since $1 \in \mathcal{W}$, it follows that

$$
\lim _{x \rightarrow \infty} x^{-1} \sum_{n \leq x} \sigma_{-s}^{*}(n)=\sum_{n=1}^{\infty} \frac{\phi(n)}{n^{s+2}}=\frac{\zeta(s+1)}{\zeta(s+2)},
$$

where $\sigma_{s}^{*}(n)$ denotes the sum of the $s$ th powers of the unitary divisors of $n$, that is,

$$
\sigma_{s}^{*}(n):=\sum_{\substack{d \mid n \\(d, n / d)=1}} d^{s}
$$

THEOREM 8. Let $f$ be a number-theoretic function and let $g$ be defined implicitly by

$$
f(n)=\sum_{\substack{d \mid n \\(d, n / d)=1}} d^{s} g(n / d)
$$

for a fixed real number $s(s>0)$. Assume that $g$ is bounded. Then, as $x \rightarrow \infty$,

$$
\sum_{n \leq x} f(n)=\frac{x^{s+1}}{s+1} \sum_{n=1}^{\infty} \frac{g(n) \phi(n)}{n^{s+2}}+O\left(R_{s}^{*}(x)\right)
$$


where

$$
R_{s}^{*}(x):=x^{s} \sum_{n \leq x} \frac{2^{\omega(n)}}{n^{s}} \ll \begin{cases}x \log x & \text { if } 0 \leq s<1, \\ x \log ^{2} x & \text { if } s=1, \\ x^{s} \zeta^{2}(s) & \text { if } s>1 .\end{cases}
$$

In particular,

$$
\sum_{n \leq x} \sigma_{s}^{*}(n)=\frac{\zeta(s+1)}{(1+s) \zeta(s+2)} x^{s+1}+O\left(R_{s}^{*}(x)\right) .
$$

TheOREM 9. Let $s$ be a fixed positive number. Let $f$ and $g$ stand for arithmetic functions defined implicitly by

$$
f(n)=\sum_{\substack{d \mid n \\(d, n / d)=1}} \mu^{2}(d) d^{s} g(n / d) .
$$

Assume that $g$ is bounded. Then

$$
\lim _{x \rightarrow \infty} \frac{1}{x^{s+1}} \sum_{n \leq x} f(n)=\frac{1}{(1+s) \zeta(2)} \sum_{n=1}^{\infty} \frac{g(n) \phi(n)}{n^{s} J(n)} .
$$

The case $s=1$ of Theorems 8 and 9 is due to Cohen (cf. [2], Theorems 4.1 and 5.1).

4. Preliminary estimates. Before we proceed to prove the theorems, we need auxiliary results. We state some of them as lemmas.

Deshouillers, Dress and Tenenbaum [6], p. 275, established a now classic result related to a class of multiplicative arithmetic functions. From their result one can easily obtain, uniformly for real $x \geq 2$ and integer $k \geq 1$, the two estimates

$$
\sum_{n \leq x} \frac{1}{\tau(n k)}=\frac{\alpha}{\sqrt{\pi}} \frac{x}{\sqrt{\log x}}\left\{u(k)+O\left(\frac{1}{\log x}\right)\right\},
$$

where $u$ and $\alpha$ are defined in Theorem 1 , and

$$
\sum_{n \leq x} \frac{1}{2^{\omega(n k)}}=\frac{\beta}{\sqrt{\pi}} \frac{x}{\sqrt{\log x}}\left\{v(k)+O\left(\frac{1}{\log x}\right)\right\},
$$

where $v$ is the multiplicative function defined by

$$
v(n):=\frac{1}{2^{\omega(n)}} \prod_{p \mid n}\left(1+\frac{1}{2 p-1}\right)=\prod_{p \mid n}\left(2-\frac{1}{p}\right)^{-1}
$$

and $\beta$ is the constant defined in Theorem 2. Clearly, $|u(n)| \leq 1$ and $|v(n)| \leq 1$.

Let $n$ be a positive integer. If $U_{n}$ and $S_{n}$ stand respectively for the set of unitary divisors and the set of squarefree divisors of $n$, we clearly have

$$
\# U_{n}=\# S_{n}=2^{\omega(n)},
$$


where $\# E$ stands for the cardinality of the set $E$. Most often, for a given arithmetic function $f$ and for certain integers $n$, we have

$$
U_{n} \neq S_{n} \quad \text { and } \quad\left(f *_{\mathrm{u}} 1\right)(n) \neq\left(f *_{\mathrm{l}} 1\right)(n)
$$

where $*_{1}$ is the restriction of Dirichlet convolution to $S_{n}$. Nevertheless, as shown in the following result, if $f$ is strongly multiplicative, then the convolutions $*_{\mathrm{u}}$ and $*_{1}$ yield the same result, in which case we have $f_{2}=f_{3}$.

LEMMA 1. Given a strongly multiplicative function $f$, the function $h:=$ $\mu f * 1$ satisfies

$$
\frac{1}{2^{\omega}}\left(f *_{\mathrm{u}} 1\right)=\frac{1}{2^{\omega}}\left(f *_{1} 1\right)=\frac{\mu h}{\tau} * 1=\frac{\mu h}{2^{\omega}} * 1=\frac{\mu h}{2^{\Omega}} * 1 .
$$

Pro of. Since $f$ is strongly multiplicative, so are

$$
f_{2}:=\frac{1}{2^{\omega}}\left(f *_{\mathrm{u}} 1\right) \quad \text { and } \quad f_{3}:=\frac{1}{2^{\omega}}\left(f *_{1} 1\right)
$$

(see De Koninck et Grah [5]), and moreover, $f_{2}(p)=f_{3}(p)=\frac{1}{2}(1+f(p))$ for all primes $p$, which proves the first equality of (4.3).

For the other equalities, set $h=\mu f * 1$ and notice that

$$
\frac{1}{2}(1+f(p))=1-\frac{1}{2}(1-f(p))=1-\frac{h(p)}{2},
$$

that is,

$$
f_{2}(p)=f_{3}(p)=1-\frac{h(p)}{\tau(p)}=1-\frac{h(p)}{2^{\omega(p)}}=1-\frac{h(p)}{2^{\Omega(p)}},
$$

and this completes the proof of Lemma 1 .

Before stating the second lemma, we recall the classic result

$$
\sum_{\substack{n \leq x \\(n, \bar{k})=1}} 1=\frac{\phi(k)}{k} x+O\left(2^{\omega(k)}\right) \quad(x \rightarrow \infty),
$$

which can easily be established using the characterization identity of the Möbius function, namely

$$
\sum_{d \mid n} \mu(d)=E(n):= \begin{cases}1 & \text { if } n=1 \\ 0 & \text { if } n>1 .\end{cases}
$$

Lemma 2. Let $k$ be a fixed positive integer and $s$ a positive number. Then

$$
\sum_{\substack{n \leq x \\(n, \bar{k})=1}} \frac{\phi_{s}(n)}{n^{s}}=\frac{\phi(k) k^{s}}{\phi_{s+1}(k)} \frac{x}{\zeta(s+1)}+O\left(R_{k, s}(x)\right),
$$


where

$$
R_{k, s}(x):=2^{\omega(k)} \sum_{\substack{d \leq x \\(d, k)=1}} \frac{|\mu(d)|}{d^{s}} \ll \begin{cases}2^{\omega(k)} \frac{\phi_{s}(k)}{k^{s}} & \text { if } s>1, \\ 2^{\omega(k)} \frac{\phi(k)}{k} \log x & \text { if } s=1, \\ 2^{\omega(k)} \frac{\phi(k)}{k} x^{1-s} & \text { if } 0<s<1 .\end{cases}
$$

Pr o of. Using (4.4), (2.3) and the definition of $R_{k, s}(x)$, we obtain

$$
\begin{aligned}
\sum_{\substack{n \leq x \\
(n, k)=1}} \frac{\phi_{s}(n)}{n^{s}} & =\sum_{\substack{d \leq x \\
(d, \bar{k})=1}} \frac{\mu(d)}{d^{s}} \sum_{\substack{m \leq x / d \\
(m, k)=1}} 1 \\
& =x \frac{\phi(k)}{k} \sum_{\substack{d \leq x \\
(d, k)=1}} \frac{\mu(d)}{d^{s+1}}+O\left(R_{k, s}(x)\right) .
\end{aligned}
$$

Now the infinite series $\sum_{n=1,(n, k)=1}^{\infty} \mu(n) / n^{s+1}$ is absolutely convergent when $s>0$ and

$$
\sum_{\substack{d \leq x \\(d, \bar{k})=1}} \frac{\mu(d)}{d^{s+1}}=\frac{k^{s+1}}{\zeta(s+1) \phi_{s+1}(k)}+O\left(x^{-s}\right) .
$$

From this it follows that

$$
\sum_{\substack{n \leq x \\(n, k)=1}} \frac{\phi_{s}(n)}{n^{s}}=\frac{k^{s} \phi(k)}{\phi_{s+1}(k)} \cdot \frac{x}{\zeta(s+1)}+O\left(\frac{\phi(k)}{k} x^{1-s}\right)+O\left(R_{k, s}(x)\right) .
$$

Now, to estimate $R_{k, s}(x)$, one can use (4.5) and the Euler-MacLaurin formula (cf. [1], p. 55), thereby obtaining

$$
\begin{array}{lll}
R_{k, 1}(x) & \ll 2^{\omega(k)} \frac{\phi(k)}{k} \log x, & \\
R_{k, s}(x) \ll 2^{\omega(k)} \frac{\phi_{s}(k)}{k^{s}} & \text { for } s>1, \\
R_{k, s}(x) \ll 2^{\omega(k)} \frac{\phi(k)}{k} x^{1-s} & \text { for } 0<s<1 .
\end{array}
$$

From these estimates we see that in each case the first $O(\ldots)$ term of $(4.9)$ is dominated by the one containing $R_{k, s}(x)$. The lemma is proved.

Lemma 3. Let $f$ be a function in $\mathcal{W}$. Then, for each fixed positive integer $k$,

$$
\sum_{\substack{n \leq x \\ n \equiv 0(\bmod k)}} f(n)=\left(a_{k}+o(1)\right) x
$$


where

$$
a_{k}=\frac{1}{k} \sum_{d \mid k} \frac{\phi(d)}{d} \sum_{m=1}^{\infty} \frac{(\mu * f)(d m)}{m} .
$$

Since $f \in \mathcal{W}$, there exists a certain function $g$ such that $f(n)=\sum_{d \mid n} g(d)$ and such that $\sum_{n=1}^{\infty}|g(n)| / n$ is convergent. By inversion this convergence is equivalent to that of the series $\sum_{n=1}^{\infty}|(\mu * f)(n)| / n$. For the proof of (4.10), we refer the reader to Theorem $1^{\prime \prime}$ in Postnikov [8], p. 142, our Lemma 3 being a particular case of that theorem.

Finally, for the last preliminary result, we have, using (4.5),

$$
\sum_{\substack{n \leq x \\(n, k)=1}} n^{s}=\sum_{n \leq x} n^{s} \sum_{r \mid(n, k)} \mu(r)=\sum_{r \mid k} \mu(r) r^{s} \sum_{m \leq x / r} m^{s} .
$$

From the Euler-MacLaurin formula, this last sum is easily estimated, and we obtain

Lemma 4. If $x \geq 2$ while $k \geq 1$ and $s \geq 0$ are fixed, then

$$
\sum_{\substack{n \leq x \\(n, \bar{k})=1}} n^{s}=\frac{x^{1+s}}{1+s} \cdot \frac{\phi(k)}{k}+O\left(2^{\omega(k)} x^{s}\right)
$$

and

$$
\sum_{\substack{n \leq x \\(n, k)=1}} \frac{1}{n^{s}}= \begin{cases}\frac{\phi_{s}(k)}{k^{s}} \zeta(s)+O\left(x^{1-s} \frac{\phi(k)}{k}\right) & \text { if } s>1, \\ \frac{\phi(k)}{k}(\log x+\gamma) & \text { if } s=1, \\ -\sum_{r \mid k} \frac{\mu(r)}{r} \log r+O\left(\frac{2^{\omega(k)}}{x}\right) & \text { if } 0 \leq s<1, \\ \frac{x^{1-s}}{1-s} \frac{\phi(k)}{k}+O\left(2^{\omega(k)} x^{-s}\right) & \text { if } 1,\end{cases}
$$

where $\gamma$ stands for Euler's constant.

\section{Proofs of the main results}

Proof of Theorem 1. Let $f$ be as in the statement of the theorem. Then

$$
\sum_{n \leq x} \frac{1}{\tau(n)} \sum_{d \mid n} f(d)=\sum_{d \leq x} f(d) \sum_{m \leq x / d} \frac{1}{\tau(m d)}
$$




$$
\begin{aligned}
& =\sum_{d \leq x / 2} f(d) \sum_{m \leq x / d} \frac{1}{\tau(m d)}+\sum_{x / 2<d \leq x} f(d) \sum_{m \leq x / d} \frac{1}{\tau(m d)} \\
& =\sum_{d \leq x / 2} f(d) \sum_{m \leq x / d} \frac{1}{\tau(m d)}+\sum_{x / 2<d \leq x} \frac{f(d)}{\tau(d)}
\end{aligned}
$$

where we have used the fact that for $d>x / 2$, i.e. $x / d<2$, the sum $\sum_{m \leq x / d} 1 / \tau(m d)$ is reduced to $1 / \tau(d)$.

Using Cauchy's inequality, we have

$$
\begin{aligned}
\sum_{x / 2<d \leq x} \frac{f(d)}{\tau(d)} & =\sum_{x / 2<d \leq x} \frac{f(d)}{\sqrt{d}} \cdot \frac{\sqrt{d}}{\tau(d)} \\
& \leq\left(\sum_{x / 2<d \leq x} \frac{f^{2}(d)}{d} \sum_{x / 2<d \leq x} \frac{d}{\tau^{2}(d)}\right)^{1 / 2} \\
& <\left(\sum_{d>x / 2} \frac{f^{2}(d)}{d} \sum_{d \leq x} \frac{d}{\tau^{2}(d)}\right)^{1 / 2} .
\end{aligned}
$$

By the classical estimate

$$
\sum_{n \leq x} \frac{1}{\tau^{2}(n)} \ll x \log ^{-3 / 4} x \quad(x \rightarrow \infty)
$$

we have

$$
\sum_{d \leq x} \frac{d}{\tau^{2}(d)} \ll x^{2} \log ^{-3 / 4} x \quad(x \rightarrow \infty) .
$$

Inserting (5.3) and condition (3.1) in (5.2) gives

$$
\sum_{x / 2<d \leq x} \frac{f(d)}{\tau(d)}=o\left(\frac{x}{\sqrt{\log x}}\right)
$$

and (5.1) becomes

$$
\sum_{n \leq x} \frac{1}{\tau(n)} \sum_{d \mid n} f(d)=\sum_{d \leq x / 2} f(d) \sum_{m \leq x / d} \frac{1}{\tau(m d)}+o\left(\frac{x}{\sqrt{\log x}}\right) .
$$

Thus (4.1) gives

$$
\begin{aligned}
\sum_{d \leq x / 2} f(d) \sum_{m \leq x / d} \frac{1}{\tau(m d)} & =\frac{x \alpha}{\sqrt{\pi}} \sum_{d \leq x / 2} \frac{f(d)}{d \sqrt{\log (x / d)}}\left(u(d)+O\left(\frac{1}{\log (x / d)}\right)\right) \\
= & \frac{x \alpha}{\sqrt{\pi}} \sum_{d \leq x / 2} \frac{f(d) u(d)}{d \sqrt{\log (x / d)}}+O\left(x \sum_{d \leq x / 2} \frac{|f(d)|}{d \log ^{3 / 2}(x / d)}\right)
\end{aligned}
$$


Now, using the convergence of the infinite series $\sum_{d=1}^{\infty}|f(d)| / d$, one obtains

$$
\begin{aligned}
x \sum_{d \leq x / 2} \frac{|f(d)|}{d \log ^{3 / 2}(x / d)} & =\frac{x}{\log ^{3 / 2} x} \sum_{d \leq x / 2} \frac{|f(d)|}{d}\left(1+O\left(\frac{\log d}{\log x}\right)\right) \\
& \ll \frac{x}{\log ^{3 / 2} x} \sum_{d \leq x / 2} \frac{|f(d)|}{d} \\
& \ll \frac{x}{\log ^{3 / 2} x}=o\left(\frac{x}{\log ^{1 / 2} x}\right) .
\end{aligned}
$$

Therefore,

$$
\begin{aligned}
& \sum_{n \leq x} \frac{1}{\tau(n)} \sum_{d \mid n} f(d) \\
& \quad=\frac{x \alpha}{\sqrt{\pi}} \sum_{d \leq x / 2} \frac{f(d) u(d)}{d \sqrt{\log (x / d)}}+o\left(\frac{x}{\log ^{1 / 2} x}\right) \\
& \quad=\frac{\alpha}{\sqrt{\pi}} \frac{x}{\sqrt{\log x}} \sum_{d \leq x / 2} \frac{f(d) u(d)}{d}\left(1+O\left(\frac{\log d}{\log x}\right)\right)+o\left(\frac{x}{\sqrt{\log x}}\right) .
\end{aligned}
$$

Using once more the convergence of $\sum_{n=1}^{\infty}|f(n)| / n$ and the estimate $|u(n)|$ $\leq 1$, we deduce the convergence of $\sum_{d=1}^{\infty} f(d) u(d) / d$. By partial summation, it follows from the convergence of $\sum_{n=1}^{\infty}|f(n)| / n$ that as $x \rightarrow \infty$,

$$
\sum_{d \leq x / 2} \frac{|f(d)|}{d} \log d=o(\log x) .
$$

Therefore, it follows that

$$
\begin{aligned}
\sum_{n \leq x} \frac{1}{\tau(n)} \sum_{d \mid n} f(d)= & \frac{\alpha}{\sqrt{\pi}} \frac{x}{\sqrt{\log x}}\left(\sum_{d=1}^{\infty} \frac{f(d) u(d)}{d}-\sum_{d>x / 2} \frac{f(d) u(d)}{d}\right) \\
& +O\left(\frac{x}{\log ^{3 / 2} x} \sum_{d \leq x / 2} \frac{|f(d)| \log d}{d}\right)+o\left(\frac{x}{\sqrt{\log x}}\right) \\
= & A \frac{x}{\sqrt{\log x}}+o\left(\frac{x}{\sqrt{\log x}}\right)+o\left(\frac{x}{\log ^{3 / 2} x} \log x\right) \\
= & (A+o(1)) \frac{x}{\sqrt{\log x}} .
\end{aligned}
$$

Theorem 1 is thus proved. 
Proof of Theorem 2. To prove (3.4), we start as follows:

$$
\begin{aligned}
\sum_{n \leq x} \frac{1}{2^{\omega(n)}} \sum_{\substack{d \mid n \\
(d, n / d)=1}} f(d)= & \sum_{d \leq x} f(d) \sum_{\substack{m \leq x / d \\
(m, d)=1}} \frac{1}{2^{\omega(m d)}} \\
= & \sum_{d \leq x^{1 / 3}} f(d) \sum_{\substack{m \leq x / d \\
(m, d)=1}} \frac{1}{2^{\omega(m d)}} \\
& +\sum_{x^{1 / 3}<d \leq x} f(d) \sum_{\substack{m \leq x / d \\
(m, d)=1}} \frac{1}{2^{\omega(m d)}},
\end{aligned}
$$

that is,

$$
\sum_{n \leq x} \frac{1}{2^{\omega(n)}} \sum_{\substack{d \mid n \\(d, n / d)=1}} f(d)=\sum_{1}+\sum_{2},
$$

say. To estimate $\sum_{2}$, we make use of Cauchy's inequality:

$$
\begin{aligned}
\sum_{2} & :=\sum_{x^{1 / 3}<d \leq x} f(d) \sum_{\substack{m \leq x / d \\
(m, d)=1}} \frac{1}{2^{\omega(m d)}} \\
& =\sum_{x^{1 / 3}<d \leq x} \frac{f(d)}{2^{\omega(d)}} \sum_{\substack{m \leq x / d \\
(m, d)=1}} \frac{1}{2^{\omega(m)}} \ll x \sum_{x^{1 / 3}<d \leq x} \frac{|f(d)|}{d 2^{\omega(d)}} \\
& =x \sum_{x^{1 / 3}<d \leq x} \frac{1}{\sqrt{d} 2^{\omega(d)}} \frac{|f(d)|}{\sqrt{d}} \ll x\left(\sum_{x^{1 / 3}<d \leq x} \frac{1}{d 4^{\omega(d)}} \sum_{x^{1 / 3}<d \leq x} \frac{f^{2}(d)}{d}\right)^{1 / 2},
\end{aligned}
$$

and therefore

$$
\sum_{2} \ll x\left(\sum_{d \leq x} \frac{1}{d 4^{\omega(d)}} \sum_{d>x^{1 / 3}} \frac{f^{2}(d)}{d}\right)^{1 / 2} .
$$

On the other hand, as $x \rightarrow \infty$,

$$
\sum_{d \leq x} \frac{1}{4^{\omega(d)}} \ll x / \log ^{3 / 4} x
$$

which combined with Abel's identity yields

$$
\sum_{d \leq x} \frac{1}{d 4^{\omega(d)}} \ll \frac{1}{\log ^{3 / 4} x}+\int_{2}^{x} \frac{d t}{t \log ^{3 / 4} t} \ll \log ^{1 / 4} x .
$$


By this estimate and (3.3), relations (5.5) and (5.4) become respectively

$$
\sum_{2}=o\left(\frac{x}{\sqrt{\log x}}\right)
$$

and

$$
\sum_{n \leq x} \frac{1}{2^{\omega(n)}} \sum_{\substack{d \mid n \\(d, n / d)=1}} f(d)=\sum_{1}+o\left(\frac{x}{\sqrt{\log x}}\right) .
$$

Now, to end the proof of (3.4) it remains to estimate $\sum_{1}$. Using (4.5) we have

$$
\begin{aligned}
\sum_{1} & =\sum_{d \leq x^{1 / 3}} f(d) \sum_{\substack{m \leq x / d \\
(m, d)=1}} \frac{1}{2^{\omega(m d)}} \\
& =\sum_{d \leq x^{1 / 3}} \frac{f(d)}{2^{\omega(d)}} \sum_{\substack{m \leq x / d \\
(m, d)=1}} \frac{1}{2^{\omega(m)}} \\
& =\sum_{d \leq x^{1 / 3}} \frac{f(d)}{2^{\omega(d)}} \sum_{m \leq x / d} \frac{1}{2^{\omega(m)}} \sum_{r \mid(d, m)} \mu(r) \\
& =\sum_{d \leq x^{1 / 3}} \frac{f(d)}{2^{\omega(d)}} \sum_{r \mid d} \mu(r) \sum_{n \leq x / d r} \frac{1}{2^{\omega(n r)}} .
\end{aligned}
$$

Since in $\sum_{1}$, we have $d \leq x^{1 / 3}$, it follows that $x^{1 / 3} \leq x /(d r) \leq x$ and therefore, using (4.2), the estimate $|v(n)| \leq 1$ and the absolute convergence of $\sum_{d=1}^{\infty} f(d) / d$, we have

$$
\begin{aligned}
\sum_{d \leq x^{1 / 3}} \frac{f(d)}{2^{\omega(d)}} & \sum_{r \mid d} \mu(r) \sum_{n \leq x / d r} \frac{1}{2^{\omega(n r)}} \\
& =\frac{x \beta}{\sqrt{\pi}} \sum_{d \leq x^{1 / 3}} \frac{f(d)}{d 2^{\omega(d)}} \sum_{r \mid d} \frac{\mu(r) v(r)}{r} \cdot \frac{1}{\sqrt{\log (x /(d r))}}+R_{1}(x),
\end{aligned}
$$

where

$$
\begin{aligned}
R_{1}(x) & \ll x \sum_{d \leq x^{1 / 3}} \frac{|f(d)|}{d 2^{\omega(d)}} \sum_{r \mid d} \frac{1}{r} \frac{1}{\log ^{3 / 2}(x /(d r))} \ll \frac{x}{\log ^{3 / 2} x} \sum_{d \leq x^{1 / 3}} \frac{|f(d)|}{d 2^{\omega(d)}} \frac{\sigma(d)}{d} \\
& <\frac{x}{\log ^{3 / 2} x} \sum_{d \leq x^{1 / 3}} \frac{|f(d)|}{d} \ll \frac{x}{\log ^{3 / 2} x},
\end{aligned}
$$

because $x^{1 / 3} \leq x /(d r) \leq x$ implies $\frac{1}{3} \log x \leq \log (x /(d r)) \leq \log x$, and we have $\sigma(d) /\left(d 2^{\omega(d)}\right)<1$. 
Finally, because

$$
\begin{aligned}
\sum_{d \leq x^{1 / 3}} \frac{f(d)}{d 2^{\omega(d)}} \sum_{r \mid d} \frac{\mu(r) v(r)}{r} & \cdot \frac{1}{\sqrt{\log (x /(d r))}} \\
= & \frac{1}{\sqrt{\log x}} \sum_{d \leq x^{1 / 3}} \frac{f(d)}{d 2^{\omega(d)}} \sum_{r \mid d} \frac{\mu(r) v(r)}{r}+R_{2}(x),
\end{aligned}
$$

where

$$
\begin{aligned}
R_{2}(x) & \ll \frac{1}{\log ^{3 / 2} x} \sum_{d \leq x^{1 / 3}} \frac{|f(d)|}{d 2^{\omega(d)}} \log d \sum_{r \mid d} \frac{1}{r}=\frac{1}{\log ^{3 / 2} x} \sum_{d \leq x^{1 / 3}} \frac{|f(d)| \sigma(d)}{d^{2} 2^{\omega(d)}} \log d \\
& <\frac{1}{\log ^{3 / 2} x} \sum_{d \leq x^{1 / 3}} \frac{|f(d)|}{d} \log d=o\left(\frac{1}{\log ^{1 / 2} x}\right)
\end{aligned}
$$

we conclude that as $x \rightarrow \infty$,

$\sum_{1}=\frac{\beta}{\sqrt{\pi}} \cdot \frac{x}{\sqrt{\log x}}\left(\sum_{d=1}^{\infty} \frac{f(d)}{d 2^{\omega(d)}} \sum_{r \mid d} \frac{\mu(r) v(r)}{r}+o(1)\right)=(B+o(1)) \frac{x}{\sqrt{\log x}}$,

where we used the multiplicativity of the function $\mu(n) v(n) / n$. Hence (3.4) follows.

The proof of (3.5) can be handled in a manner similar to that of (3.4), by simply using the equality

$$
\begin{aligned}
\sum_{n \leq x} \frac{1}{2^{\omega(n)}} \sum_{\substack{d \mid n \\
d \text { squarefree }}} f(d)= & \sum_{d \leq x / 2} \mu^{2}(d) f(d) \sum_{m \leq x / d} \frac{1}{2^{\omega(m d)}} \\
& +\sum_{x / 2<d \leq x} \frac{\mu^{2}(d) f(d)}{2^{\omega(d)}}
\end{aligned}
$$

In fact, from (3.3) and Cauchy's inequality, we conclude that as $x \rightarrow \infty$,

$$
\sum_{x / 2<d \leq x} \frac{\mu^{2}(d) f(d)}{2^{\omega(d)}}=o\left(\frac{x}{\sqrt{\log x}}\right) .
$$

Hence, using (4.2), one completes the proof.

Remarks. 1. Theorem 1 is based on two conditions: the absolute convergence of the infinite series $\sum_{n=1}^{\infty} f(n) / n$, and the inequality

$$
\sum_{n>x} \frac{f^{2}(n)}{n}=o\left(\frac{1}{\log ^{1 / 4} x}\right) \text {. }
$$

Most likely, the second condition is not necessary, but we could not prove 
the result without it. In fact, to this end, one should prove that, as $x \rightarrow \infty$,

$$
\sum_{x / 2<n \leq x} \frac{f(n)}{\tau(n)}=o\left(\frac{x}{\sqrt{\log x}}\right), \quad \text { if } \sum_{n=1}^{\infty} \frac{|f(n)|}{n} \text { converges. }
$$

The same holds for Theorem 2 and condition (3.3).

2. By assuming the absolute convergence of the three series defining the constants $A, B$ and $D$ instead of that of $\sum_{n=1}^{\infty} f(n) / n$, the conclusions of Theorems 1 and 2 can clearly be restated with weaker hypothesis.

Proof of Theorem 3. As shown in De Koninck and Grah [5], $f_{3}$ is strongly multiplicative, since $f$ is multiplicative. Thus it follows from (4.3) that

$$
f_{3}(n):=\frac{1}{2^{\omega(n)}} \sum_{\substack{d \mid n \\ d \text { squarefree }}} f(d)=\sum_{d \mid n} \frac{\mu(d) h(d)}{\tau(d)} .
$$

Since $\sum_{n=1}^{\infty} \mu^{2}(n)|h(n)| /(n \tau(n))$ converges, (3.6) is proved. The last conclusion of the theorem is clearly a consequence of the fact that $f_{2} \equiv f_{3}$ when $f$ is strongly multiplicative.

Proof of Theorem 4 . We investigate only the case $s \neq 0$. Indeed, the case $s=0$ is proved as Proposition 4 in [4], which we mentioned in the remarks following the statement of Theorem 4.

From (2.1) we have

$$
\sum_{n \leq x} f(n)=\sum_{n \leq x} \frac{1}{n^{s}} \sum_{d \mid n} \bar{f}_{s}(d) \phi_{s}(d)=\sum_{d \leq x} \frac{\bar{f}_{s}(d) \phi_{s}(d)}{d^{s}} R_{s}(x / d),
$$

where

$$
R_{s}(x):=\sum_{n \leq x} 1 / n^{s} .
$$

In a manner similar to that used to evaluate $R_{k, s}(x)$ in (4.7), one can prove that the following expansions are valid:

$$
R_{s}(x)=\sum_{n \leq x} \frac{1}{n^{s}}= \begin{cases}\zeta(s)+O\left(1 / x^{s-1}\right) & \text { if } s>1, \\ \log x+\gamma+O(1 / x) & \text { if } s=1, \\ x^{1-s} /(1-s)+O\left(x^{-s}\right) & \text { if } 0<s<1, \\ x^{r+1} /(1+r)+O\left(x^{r}\right) & \text { if } r=-s>0\end{cases}
$$

If for $s=1, \sum_{n=1}^{\infty}\left|\bar{f}_{1}(n)\right| \phi(n) / n$ is convergent, then using the second relation of (5.6), we have

$$
\sum_{n \leq x} f(n)=\sum_{d \leq x} \frac{\bar{f}_{1}(d) \phi(d)}{d}\{\log (x / d)+\gamma+O(d / x)\}
$$




$$
\begin{aligned}
= & (\log x+\gamma) \sum_{d \leq x} \frac{\bar{f}_{1}(d) \phi(d)}{d} \\
& -\sum_{d \leq x} \frac{\bar{f}_{1}(d) \phi(d)}{d} \log d+O\left(x^{-1} \sum_{d \leq x}\left|\bar{f}_{1}(d)\right| \phi(d)\right) \\
= & (\log x+\gamma)\left(c_{1}+o(1)\right) \\
& -\sum_{d \leq x} \frac{\bar{f}_{1}(d) \phi(d)}{d} \log d+O\left(x^{-1} \sum_{d \leq x}\left|\bar{f}_{1}(d)\right| \phi(d)\right) .
\end{aligned}
$$

To establish (3.8), we must show that

$$
\sum_{d \leq x} \frac{\bar{f}_{1}(d) \phi(d)}{d} \log d \quad \text { and } \quad x^{-1} \sum_{d \leq x} \bar{f}_{1}(d) \phi(d)
$$

are both $o(\log x)$. Indeed using the convergence of $\sum_{n=1}^{\infty}\left|\bar{f}_{1}(n)\right| \phi(n) / n$ and partial summation, we obtain

$$
\sum_{d \leq x} \frac{\left|\bar{f}_{1}(d)\right| \phi(d)}{d} \log d=o(\log x) \quad \text { and } \quad x^{-1} \sum_{d \leq x}\left|\bar{f}_{1}(d)\right| \phi(d)=o(1) .
$$

Similarly, the other three results of Theorem 4 are derived by repeated use of partial summation and absolute convergence of the indicated series.

Proof of Theorem 5. If $f(n)$ is defined as in (2.2), we obtain

$$
\begin{aligned}
\sum_{n \leq x} f(n) & =\sum_{d \leq x} \frac{\widetilde{f}_{s}(d)}{d^{s} \phi_{s}(\delta(d))} \sum_{\substack{m \leq x / d \\
(m, d)=1}} \frac{\phi_{s}(d m)}{m^{s}} \\
& =\sum_{d \leq x} \frac{\widetilde{f}_{s}(d) \phi_{s}(d)}{d^{s} \phi_{s}(\delta(d))} \sum_{\substack{m \leq x / d \\
(m, d)=1}} \frac{\phi_{s}(m)}{m^{s}} .
\end{aligned}
$$

By using Lemma 2 and the simplification

$$
\frac{\phi_{s}(n)}{n \phi_{s}(\delta(n))}=\frac{n^{s-1}}{\delta(n)^{s}}
$$

we find that

$$
\begin{aligned}
\sum_{n \leq x} f(n) & =\frac{x}{\zeta(s+1)} \sum_{d \leq x} \frac{\widetilde{f}_{s}(d) \phi(d) \phi_{s}(d)}{d \phi_{s}(\delta(d)) \phi_{s+1}(d)}+O\left(\sum_{d \leq x} \frac{\left|\tilde{f}_{s}(d)\right| \phi_{s}(d)}{d^{s} \phi_{s}(\delta(d))} R_{d, s}(x / d)\right) \\
& =\frac{x}{\zeta(s+1)} \sum_{d \leq x} \frac{\widetilde{f}_{s}(d) \phi(d) d^{s-1}}{\delta(d)^{s} \phi_{s+1}(d)}+O\left(\sum_{d \leq x} \frac{\left|\tilde{f}_{s}(d)\right|}{\delta(d)^{s}} R_{d, s}(x / d)\right) .
\end{aligned}
$$


Furthermore, we have (see the Remarks following Theorem 5)

$$
\begin{aligned}
\sum_{n \leq x} f(n)= & \frac{x}{\zeta(s+1)} \sum_{d=1}^{\infty} \frac{\widetilde{f}_{s}(d) \phi(d) d^{s-1}}{\delta(d)^{s} \phi_{s+1}(d)}+o(x) \\
& +O\left(\sum_{d \leq x} \frac{\left|\widetilde{f}_{s}(d)\right|}{\delta(d)^{s}} R_{d, s}(x / d)\right) .
\end{aligned}
$$

To complete the proof we need to establish the following:

$$
\sum_{d \leq x} \frac{\left|\widetilde{f}_{s}(d)\right|}{\delta(d)^{s}} R_{d, s}(x / d)=o(x) \quad \text { for all } s>0 .
$$

Because of (4.7) and of the boundedness of $\widetilde{f}_{s}$, we have

$$
\sum_{d \leq x} \frac{\left|\widetilde{f}_{s}(d)\right|}{\delta(d)^{s}} R_{d, s}(x / d) \ll \sum_{d \leq x} \frac{2^{\omega(d)}}{\delta(d)^{s}} \quad(s>1),
$$

and

$$
\sum_{d \leq x} \frac{\left|\widetilde{f}_{1}(d)\right|}{\delta(d)} R_{d, 1}(x / d) \ll \sum_{d \leq x} \frac{2^{\omega(d)}}{\delta(d)} \log (x / d) \ll \log x \sum_{d \leq x} \frac{2^{\omega(d)}}{\delta(d)} .
$$

Likewise, since the series

$$
\sum_{n=1}^{\infty} \frac{2^{\omega(n)}}{\sqrt{n} \delta(n)^{s}}=\prod_{p}\left(1+\frac{2}{p^{s}\left(p^{1 / 2}-1\right)}\right)
$$

is convergent for $s>1 / 2$ we have, using partial summation,

$$
\sum_{d \leq x} \frac{2^{\omega(d)}}{\delta(d)^{s}}=\sum_{d \leq x} \sqrt{d} \frac{2^{\omega(d)}}{\delta(d)^{s} \sqrt{d}}=o(\sqrt{x}) .
$$

This proves (3.12) for $s \geq 1$.

Using once more partial summation with $0<s<1$ yields

$$
\sum_{d \leq x} \frac{\left|\widetilde{f}_{s}(d)\right|}{\delta(d)^{s}} R_{d, s}(x / d) \ll x^{1-s} \sum_{d \leq x} \frac{2^{\omega(d)}}{d^{1-s} \delta(d)^{s}}=x^{1-s} \sum_{d \leq x} d^{s} \frac{2^{\omega(d)}}{\delta(d)^{s} d}=o(x),
$$

since clearly $\sum_{d=1}^{\infty} 2^{\omega(d)} /\left(\delta(d)^{s} d\right)$ is convergent. This establishes the validity of (3.12) for $0<s<1$, and Theorem 5 is proved.

Proof of Theorem 6. From (3.13) one obtains

$$
\sum_{n \leq x} f(n)=\sum_{d \leq x} h(d) \sum_{\substack{m \leq x / d \\(m, d)=1}} g(m)=\sum_{d \leq x} h(d) \sum_{m \leq x / d} g(m) \sum_{r \mid(m, d)} \mu(r) .
$$


Rewriting this gives

$$
\begin{aligned}
\sum_{n \leq x} f(n) & =\sum_{d \leq x} h(d) \sum_{m \leq x / d} g(m) \sum_{r \mid(m, d)} \mu(r) \\
& =\sum_{d \leq x} h(d) \sum_{r \mid d} \mu(r) \sum_{\substack{n \leq x / d \\
n \equiv 0(\bmod r)}} g(n) .
\end{aligned}
$$

Applying Lemma 3 to this last sum, we have

$$
\begin{aligned}
\sum_{n \leq x} f(n)= & x \sum_{d \leq x} \frac{h(d)}{d} \sum_{r \mid d} \frac{\mu(r)}{r} \sum_{l \mid r} \frac{\phi(l)}{l} \sum_{m=1}^{\infty} \frac{(\mu * g)(l m)}{m} \\
& +o\left(x \sum_{d \leq x} \frac{|h(d)|}{d} \sum_{r \mid d} \mu(r)\right) .
\end{aligned}
$$

Moreover, for $d$ fixed,

$$
\begin{aligned}
\sum_{r \mid d} \frac{\mu(r)}{r} \sum_{l \mid r} \frac{\phi(l)}{l} \sum_{m=1}^{\infty} & \frac{(\mu * g)(l m)}{m} \\
& \ll\left|\sum_{r \mid d} \frac{\mu(r)}{r} \sum_{l \mid r} \frac{\phi(l)}{l}\right| \sum_{m=1}^{\infty} \frac{|(\mu * g)(l m)|}{m} \\
& \ll \sum_{r \mid d} \frac{\mu(r)}{r} \sum_{l \mid r} \frac{\phi(l)}{l}=\prod_{p \mid d}\left(1-\frac{1}{p}\right)^{2}<1,
\end{aligned}
$$

where we used the fact that $g \in \mathcal{W}$.

Furthermore, from (5.4) and the convergence of $\sum_{d=1}^{\infty}|h(d)| / d$, we deduce that

$$
\begin{aligned}
& \sum_{d>x} \frac{h(d)}{d} \sum_{r \mid d} \frac{\mu(r)}{r} \sum_{l \mid r} \frac{\phi(l)}{l} \sum_{m=1}^{\infty} \frac{(\mu * g)(l m)}{m} \\
&+o\left(\sum_{d \leq x} \frac{|h(d)|}{d} \sum_{r \mid d} \mu(r)\right)=o(1) .
\end{aligned}
$$

Thus, if we take into account (5.9) and (5.10), Theorem 6 follows immediately.

Corollary 7 is an immediate consequence of Theorem 6 .

Proofs of Theorems 8 and 9. Since (3.16) and (3.18) are particular cases of (3.13), we can use (5.8) to prove (3.17) and (3.19). 
Indeed for $s \geq 0$, adapting (5.8) to the summatory function of (3.16) gives

$$
\sum_{n \leq x} f(n)=\sum_{n \leq x} \sum_{\begin{array}{c}
d \mid n \\
(d, n / d)=1
\end{array}} d^{s} g(n / d)=\sum_{d \leq x} g(d) \sum_{r \mid d} \mu(r) r^{s} \sum_{m \leq x /(d r)} m^{s} .
$$

Thus, Lemma 4 applied to this sum yields

$$
\sum_{n \leq x} f(n)=\frac{x^{s+1}}{s+1} \sum_{d \leq x} \frac{g(d) \phi(d)}{d^{s+2}}+O\left(x^{s} \sum_{d \leq x} \frac{|g(d)| 2^{\omega(d)}}{d^{s}}\right) .
$$

Now, using the boundedness of $g$ combined with the estimate

$$
\sum_{d \leq x} \frac{2^{\omega(d)}}{d^{s}} \leq \sum_{d \leq x} \frac{1}{d^{s}} \sum_{r \mid d} 1=\sum_{r \leq x} \frac{1}{r^{s}} \sum_{m \leq x / r} \frac{1}{m^{s}},
$$

which is deduced from Lemma 4, one easily obtains (3.17).

We now prove (3.19). First, (5.8) applied to the summatory function of (3.18) gives

$$
\begin{aligned}
\sum_{n \leq x} f(n) & =\sum_{n \leq x} \sum_{\begin{array}{c}
d \mid n \\
(d, n / d)=1
\end{array}} \mu^{2}(d) d^{s} g(n / d) \\
& =\sum_{m \leq x} g(m) \sum_{\substack{r^{2} \leq x / m \\
(r, m)=1}} \mu(r) r^{2 s} \sum_{\substack{d \leq x /\left(m r^{2}\right) \\
(d, m)=1}} d^{s} .
\end{aligned}
$$

By Lemma 4, this sum can be written as

$$
\begin{aligned}
\sum_{n \leq x} f(n)= & \frac{x^{s+1}}{s+1} \sum_{m \leq x} \frac{g(m) \phi(m)}{m^{s+2}}\left(\sum_{\substack{r=1 \\
(r, m)=1}}^{\infty} \frac{\mu(r)}{r^{2}}-\sum_{\substack{r^{2}>x / m \\
(r, m)=1}} \frac{\mu(r)}{r^{2}}\right) \\
& +O\left(x^{s} \sum_{r^{2} \leq x}|\mu(r)| \sum_{\substack{m \leq x / r^{2} \\
(m, r)=1}} \frac{2^{\omega(m)}}{m^{s}}\right),
\end{aligned}
$$

where we used the boundedness of $g$. The sequel of the proof is carried out similarly to that of (3.17), using Lemma 4, the boundedness of $g$ and the estimates

$$
\begin{gathered}
\sum_{\substack{m \leq x / r^{2} \\
(m, r)=1}} \frac{2^{\omega(m)}}{m^{s}} \ll \frac{x^{1-s} \log x}{r^{2(1-s)}}, \quad \sum_{\substack{n=1 \\
(n, k)=1}}^{\infty} \frac{\mu(n)}{n^{2}}=\frac{k^{2}}{\zeta(2) J(k)}, \\
\frac{\phi(n)}{n^{s} J(n)} \leq \frac{1}{n^{s+1}} .
\end{gathered}
$$




\section{REFERENCES}

[1] T. M. A postol, Introduction to Analytic Number Theory, Springer, New York, 1976.

[2] E. Cohen, Arithmetical functions associated with the unitary divisors of an integer, Math. Z. 74 (1960), 66-80.

[3] -, Arithmetical notes, I. On a theorem of van der Corput, Proc. Amer. Math. Soc. 12 (1961), 214-217.

[4] J. G. van der Corput, Sur quelques fonctions arithmétiques élémentaires, Proc. Konink. Nederl. Akad. Wetensch. Amsterdam 42 (1939), 859-866.

[5] J.-M. De Koninck et J. Grah, Moyennes sur certains ensembles de diviseurs d'un entier, Enseign. Math. 42 (1996), 97-123.

[6] J.-M. Deshouillers, F. Dress et G. Tenenbaum, Lois de répartition des diviseurs, 1, Acta Arith. 34 (1979), 273-285.

[7] W. Narkiewicz, On a summation formula of E. Cohen, Colloq. Math. 11 (1963), $85-86$.

[8] A. G. Postnikov, Introduction to Analytic Number Theory, Transl. Math. Monographs 68, Amer. Math. Soc., Providence, 1987.

[9] A. Wintner, Eratosthenian Averages, Waverly Press, Baltimore, 1943.

Département de mathématiques et de statistique

Université Laval, Sainte-Foy

Québec G1K 7P4

Canada

E-mail: jmdk@mat.ulaval.ca

Received 8 September 1997;

revised 15 September 1998 